\title{
Positioning Measurements of Two Industrial Robots
}

\author{
Ákos Cservenák, Tamás Szabó* \\ University of Miskolc, Robert Bosch Department of Mechatronics, H-3515, Miskolc-Egyetemváros, Hungary
}

\begin{abstract}
This paper deals with the positioning accuracies of two industrial robots, i.e., a KUKA KR 15/2 and a Fanuc LR Mate 200iC comparing measurement and modeling. The accuracy measurements are performed by Renishaw XL-80 type laser measurement system. Both robots are Articulated Manipulator (RRR), and their descriptions of Denavit-Hartenberg parameters are very similar. The inverse kinematics of the two industrial robots are also solved and analytic formulae are derived. The measurements are carried out along two perpendicular straight lines and the results are compared.
\end{abstract}

Keywords: Robot, simulation, measurement, inverse kinematics, Denavit-Hartenberg parameters.

\section{Introduction}

Nowadays robots are used even for humanoid purposes [1] in addition to industrial ones, e.g., assembly, painting, welding, workpieces handling and machining. The positioning accuracy of the robots in the later case, i.e., in machining, is a crucial question [2].

The Institute of Machine Tools and Mechatronics, in University of Miskolc, have two industrial robots, one of them is a 20 years old KUKA KR 15/2 robot and the other one is a 5 years old Fanuc LR Mate 200iC robot. Before their application to machining, it is advisable to check the accuracy of positioning. One of the best measurement device is the laser measurement system, such method was also used in [3]. This optical method is well applicable for measurements along straight line motions.

In Section 2 the kinematical description with the Denavit-Hartenberg (DH) parameters and the inverse kinematics are given for the two industrial robots ([4], [5], [6]). Measurements with laser measurement system are detailed and demonstrated in Section 3 ([7], [8]). Some concluding remarks are drawn in Section 4.

\section{Kinematical description of the robots}

The two investigated industrial robots have very similar structures and both of them can be analyzed by $\mathrm{DH}$ description. According to [6] there are four $\mathrm{DH}$ parameters, i.e., the link length $a_{\mathrm{k}^{\prime}}$ link twist $\alpha_{\mathrm{k}^{\prime}}$ link offset $s_{\mathrm{k}}$ and joint angle $\theta_{\mathrm{k}^{\prime}}$ which are defined as follows:

- $a_{\mathrm{k}}$ is the distance between the axis $z_{\mathrm{k}-1}$ and $z_{\mathrm{k}^{\prime}}$ and is measured along the axis $x_{\mathrm{k}}$

- $a_{\mathrm{k}}$ is the angle between the axis $z_{\mathrm{k}-1}$ and $z_{\mathrm{k}}$

- $s_{\mathrm{k}}$ is the distance from origin $o_{\mathrm{k}-1}$ to the intersection of the $x_{\mathrm{k}}$ axis with $z_{\mathrm{k}-1}$ measured 
along the $z_{\mathrm{k}-1}$ axis

- $\theta_{\mathrm{k}}$ is the angle from $x_{\mathrm{k}-1}$ to $x_{\mathrm{k}}$ in a plane normal to $z_{\mathrm{k}-1}$

The applied coordinate systems for the robots, i.e., KUKA KR 15/2 and Fanuc LR Mate 200iC are shown in Fig. 1a and Fig. 1b, respectively, and the DH parameters are listed in Table 1 based on [4] and [5].

The following homogenous transformation can be written between two consecutive $(k-1, k)$ coordinate systems:

$$
\begin{aligned}
\boldsymbol{H}_{k-1, k} & =\left[\begin{array}{cccc}
\boldsymbol{h}_{k-1, k} & \boldsymbol{r} \\
0 & 0 & 0 & 1
\end{array}\right] \\
& =\left[\begin{array}{cccc}
c \theta_{k} & -s \theta_{k} c \alpha_{k} & c \theta_{k} s \alpha_{k} & a_{k} c \theta_{k} \\
\theta_{k} & c \theta_{k} c \alpha_{k} & -c \theta_{k} s \alpha_{k} & a_{k} s \theta_{k} \\
0 & s \alpha_{k} & c \alpha_{k} & s_{k} \\
0 & 0 & 0 & 1
\end{array}\right]
\end{aligned}
$$

where $s \theta_{k}, c \theta_{k}$ and $s \alpha_{k}, c \alpha_{k}$ denotes sine and cosine of $\theta_{k}$ and $\alpha_{k}$, respectively.

\begin{tabular}{|c|c|c|c|c|c|c|c|c|}
\hline \multirow{2}{*}{$\begin{array}{r}\text { Parameters } \\
\text { Joints }\end{array}$} & \multicolumn{2}{|l|}{$s_{k}[\mathrm{~mm}]$} & \multicolumn{2}{|l|}{$\boldsymbol{\theta}_{k}\left[^{\circ}\right]$} & \multicolumn{2}{|l|}{$a_{k}\left[^{\circ}\right]$} & \multicolumn{2}{|c|}{$a_{k}[\mathrm{~mm}]$} \\
\hline & KUKA & Fanuc & KUKA & Fanuc & KUKA & Fanuc & KUKA & Fanuc \\
\hline J1 & 675 & 330 & $0^{\circ}$ & $0^{\circ}$ & $+90^{\circ}$ & $+90^{\circ}$ & 300 & 75 \\
\hline$J 2$ & 0 & 0 & $+180^{\circ}$ & $+90^{\circ}$ & $0^{\circ}$ & $0^{\circ}$ & 650 & 300 \\
\hline $\mathrm{J} 3$ & 0 & 0 & $0^{\circ}$ & $0^{\circ}$ & $+90^{\circ}$ & $+90^{\circ}$ & 155 & 75 \\
\hline$J 4$ & 600 & 320 & $0^{\circ}$ & $0^{\circ}$ & $-90^{\circ}$ & $-90^{\circ}$ & 0 & 0 \\
\hline$J 5$ & 0 & 0 & $0^{\circ}$ & $0^{\circ}$ & $+90^{\circ}$ & $+90^{\circ}$ & 0 & 0 \\
\hline J6 & 140 & 140 & $0^{\circ}$ & $0^{\circ}$ & $0^{\circ}$ & $0^{\circ}$ & 0 & 0 \\
\hline
\end{tabular}

Table 1: DH parameters of robots KUKA KR 15/2 and Fanuc LR Mate 200iC.

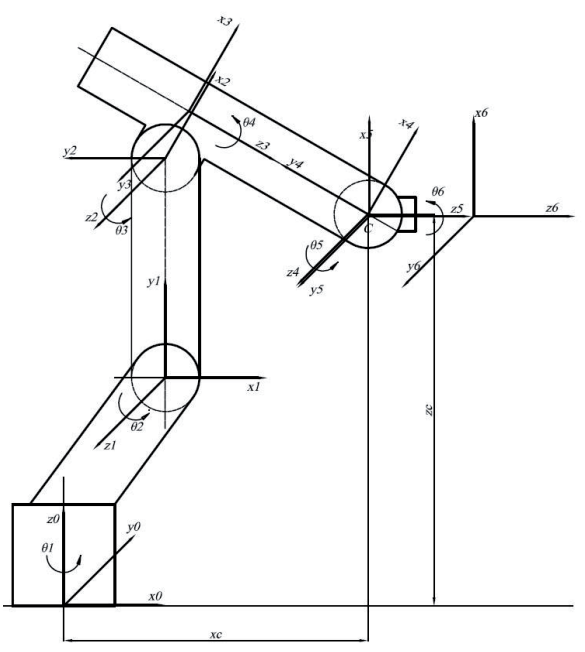

(a)

Fig. 1: Coordinate systems of (a) KUKA robot and (b) Fanuc robot.

The inverse kinematics of the robots are analyzed in order to determine the accurate values of the joint angles $\left(\theta_{k}\right)$ in measured points along prescribed straight lines. It is supposed that the orientation and path of the end effector are given. The angles of the first 3 joints give the position $\left(x_{c}, y_{c}, z_{c}\right)$ of the end effector, while the other 3 angles of the joints determine orientation of the

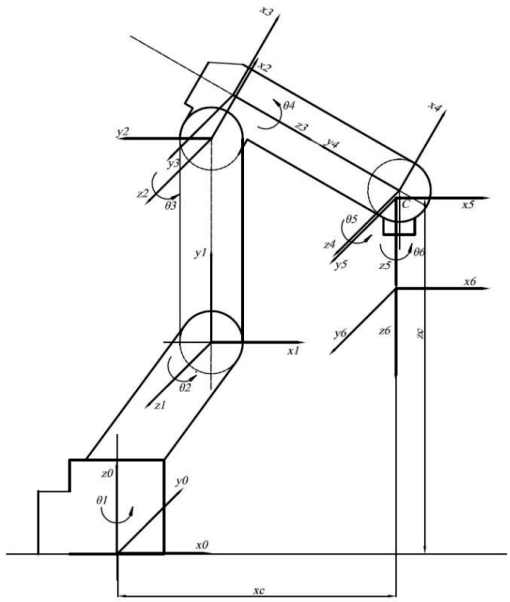

(b)

end effector.

The angle $\theta_{1}$ is shown in Fig. 2, its value can be obtained by the help of $x_{c}$ and $y_{c}$ :

$\theta_{1}=\arctan 2\left(y_{c}, x_{c}\right)$

where $\arctan 2$ provides values in interval $(-\pi,+\pi)$.

Joint angles $\theta_{2}$ and $\theta_{3}$ are shown in Fig. 3 . 


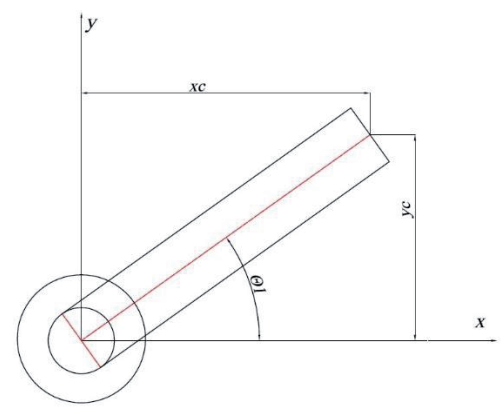

Fig. 2: Over view of the robot.

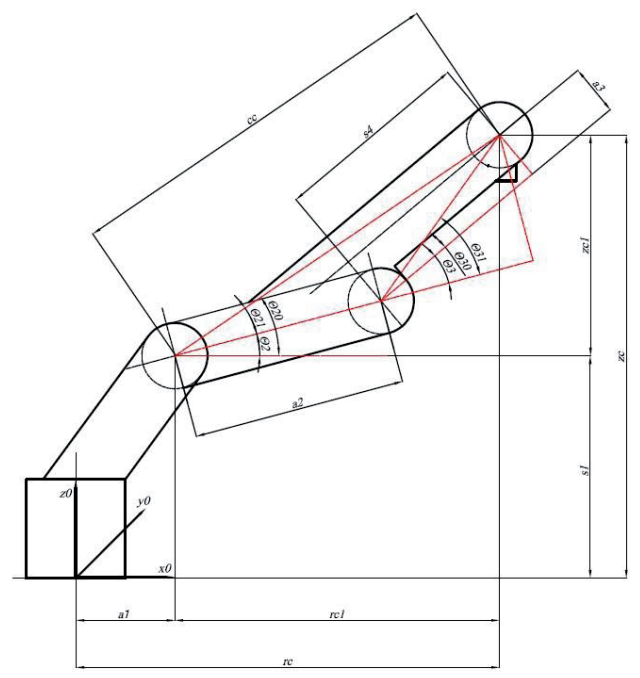

Fig. 3: Side view of the robot showing $\theta_{2}$ and $\theta_{3}$.

Distance parameters $a_{1}, a_{2}, a_{3}, s_{1}, s_{4}, z_{\mathrm{c}}, r_{\mathrm{c}}, z_{c 1}$ and $r_{c 1}$ shown in Fig. 3 are given while the last of them can be determined as follows:

$$
\begin{aligned}
& r_{c}=\sqrt{x_{c}^{2}+y_{c}^{2}} \\
& z_{c 1}=z_{c}-s_{1} \\
& r_{c 1}=r_{c}-a_{1}
\end{aligned}
$$

The joint angle $\theta_{2}$ and $\theta_{3}$ can be also obtained from simple trigonometry [6] using cosine theorem:

$$
\begin{aligned}
\theta_{3}=\theta_{30}-\theta_{31} & =\arctan 2\left( \pm \sqrt{1-D^{2}}, D\right) \\
& -\arctan 2\left(a_{3}, s_{4}\right)
\end{aligned}
$$

where

$$
D=\frac{c c^{2}-a_{2}^{2}-\left(\sqrt{s_{4}^{2}+a_{3}^{2}}\right)^{2}}{2 \cdot a_{2} \cdot \sqrt{s_{4}^{2}+a_{3}^{2}} \cdot \cos \left(180^{\circ}-\theta_{30}\right)}
$$

and

$$
\begin{aligned}
\theta_{2} & =\theta_{20}-\theta_{21} \\
& =\arctan 2\left(z_{c}-s_{1}, \sqrt{x_{c}^{2}+y_{c}^{2}}-a_{1}\right) \\
& -\arctan 2\left(\begin{array}{l}
\sqrt{s_{4}^{2}+a_{3}^{2}} \cdot \sin \left(\theta_{30}\right), \\
a_{2}+\sqrt{s_{4}^{2}+a_{3}^{2}} \cdot \cos \left(\theta_{30}\right)
\end{array}\right)
\end{aligned}
$$

where

$\theta_{30}=\arccos (D)=\arctan 2\left( \pm \sqrt{1-D^{2}}, D\right)$

Joint angles $\theta_{4}, \theta_{5}$ and $\theta_{6}$ can be obtained using transformation matrix $\boldsymbol{R}$ given by Euler angles assuming downward vertical orientation of the end effector as shown in Fig. 1 b:

$$
\boldsymbol{R}=\left[\begin{array}{lll}
r_{11} & r_{12} & r_{13} \\
r_{21} & r_{22} & r_{23} \\
r_{31} & r_{32} & r_{33}
\end{array}\right]=\left[\begin{array}{ccc}
1 & 0 & 0 \\
0 & -1 & 0 \\
0 & 0 & -1
\end{array}\right]
$$

Transformation matrix $\boldsymbol{R}$ in (9) is equal to $\boldsymbol{h}_{0,6}$ matrix which is a portion of the $\boldsymbol{H}_{0,6}$ homogoneus transformation matrix [6]:

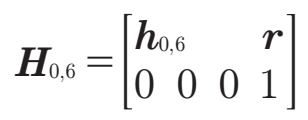

The transformation matrix $\boldsymbol{h}_{3,6}$ between the $3^{\text {rd }}$ and $6^{\text {th }}$ coordinate systems can be expressed as follows:

$$
\boldsymbol{h}_{3,6}=\left(\boldsymbol{h}_{0,3}\right)^{T} \cdot \boldsymbol{R}=\left[\begin{array}{ccc}
\cos \left(\theta_{1}\right) \cdot \cos \left(\theta_{2}+\theta_{3}\right) & -\sin \left(\theta_{1}\right) \cdot \cos \left(\theta_{2}+\theta_{3}\right) & -\sin \left(\theta_{2}+\theta_{3}\right) \\
\sin \left(\theta_{1}\right) & \cos \left(\theta_{1}\right) & 0 \\
\cos \left(\theta_{1}\right) \cdot \sin \left(\theta_{2}+\theta_{3}\right) & -\sin \left(\theta_{1}\right) \cdot \sin \left(\theta_{2}+\theta_{3}\right) & \cos \left(\theta_{2}+\theta_{3}\right)
\end{array}\right]
$$

where

$$
\left(\boldsymbol{h}_{0,3}\right)^{T}=\left[\begin{array}{ccc}
\cos \left(\theta_{1}\right) \cdot \cos \left(\theta_{2}+\theta_{3}\right) & \sin \left(\theta_{1}\right) \cdot \cos \left(\theta_{2}+\theta_{3}\right) & \sin \left(\theta_{2}+\theta_{3}\right) \\
\sin \left(\theta_{1}\right) & -\cos \left(\theta_{1}\right) & 0 \\
\cos \left(\theta_{1}\right) \cdot \sin \left(\theta_{2}+\theta_{3}\right) & \sin \left(\theta_{1}\right) \cdot \sin \left(\theta_{2}+\theta_{3}\right) & \cos \left(\theta_{2}+\theta_{3}\right)
\end{array}\right]
$$


The transformation matrix $\boldsymbol{h}_{3,6}$ in (12) can be also defined by the multiplication of three consecutive relative transformation matrices:

$$
\begin{aligned}
h_{3,6} & =\prod_{i=4 i-1, i}^{6} \\
& =\left[\begin{array}{ccc}
\mathrm{c}\left(\theta_{4}\right) \cdot \mathrm{c}\left(\theta_{5}\right) \cdot \mathrm{c}\left(\theta_{6}\right)-\mathrm{s}\left(\theta_{4}\right) \cdot \mathrm{s}\left(\theta_{6}\right) & -\mathrm{c}\left(\theta_{4}\right) \cdot \mathrm{c}\left(\theta_{5}\right) \cdot \mathrm{s}\left(\theta_{6}\right)-\mathrm{s}\left(\theta_{4}\right) \cdot \mathrm{c}\left(\theta_{6}\right) & \mathrm{c}\left(\theta_{4}\right) \cdot \mathrm{s}\left(\theta_{5}\right) \\
\mathrm{s}\left(\theta_{4}\right) \cdot \mathrm{c}\left(\theta_{5}\right) \cdot \mathrm{c}\left(\theta_{6}\right)+\mathrm{c}\left(\theta_{4}\right) \cdot \mathrm{s}\left(\theta_{6}\right) & -\mathrm{s}\left(\theta_{4}\right) \cdot \mathrm{c}\left(\theta_{5}\right) \cdot \mathrm{s}\left(\theta_{6}\right)+\mathrm{c}\left(\theta_{4}\right) \cdot \mathrm{c}\left(\theta_{6}\right) & \mathrm{s}\left(\theta_{4}\right) \cdot \mathrm{s}\left(\theta_{5}\right) \\
-\mathrm{s}\left(\theta_{5}\right) \cdot \mathrm{c}\left(\theta_{6}\right) & \mathrm{s}\left(\theta_{5}\right) \cdot \mathrm{s}\left(\theta_{6}\right) & \mathrm{c}\left(\theta_{5}\right)
\end{array}\right]
\end{aligned}
$$

Comparing the $3^{\text {rd }}$ columns and $3^{\text {rd }}$ rows of (12) and (14) one can obtained the following three equations:

$$
\begin{aligned}
& \cos \left(\theta_{5}\right)=\cos \left(\theta_{2}+\theta_{3}\right) \\
& \sin \left(\theta_{4}\right) \cdot \sin \left(\theta_{5}\right)=0 \\
& \frac{\sin \left(\theta_{5}\right) \cdot \sin \left(\theta_{6}\right)}{-\sin \left(\theta_{5}\right) \cdot \cos \left(\theta_{6}\right)} \\
& =\frac{-\sin \left(\theta_{1}\right) \cdot \sin \left(\theta_{2}+\theta_{3}\right)}{\cos \left(\theta_{1}\right) \cdot \sin \left(\theta_{2}+\theta_{3}\right)}
\end{aligned}
$$

and the solutions for $\theta_{4}, \theta_{5}$ and $\theta_{6}$ :

$$
\begin{aligned}
& \theta_{4}=0 \\
& \theta_{5}=\theta_{2}+\theta_{3} \\
& \theta_{6}=\theta_{1}
\end{aligned}
$$

\section{Measurement of the positioning}

Fig. $4 a$ shows the measurement setup for the KUKA robot while Fig. 4b illustrates the measurement setup for the Fanuc robot, where No. 1 is the linear reflector, No. 2 is the linear interferometer with linear reflector and No. 3 is the laser head ([7]). The linear reflector denoted by No. 1 is moved by the end effector, while the interferometer No. 2 and laser head No. 3 are in a static positions. The resolution of the system is $0.001 \mu \mathrm{m}[8]$.

Due to technical reasons both in the starting position and during the measurement the end effector of the KUKA robot is kept in horizontal direction, while in case of Fanuc robot the end effector is kept in vertical direction, as it is shown in Fig. 1 and Fig. 4. The joint angles of the robots in the starting position are given in Table 2 .

During the measurements of the robots the end effectors are moved horizontally firstly along
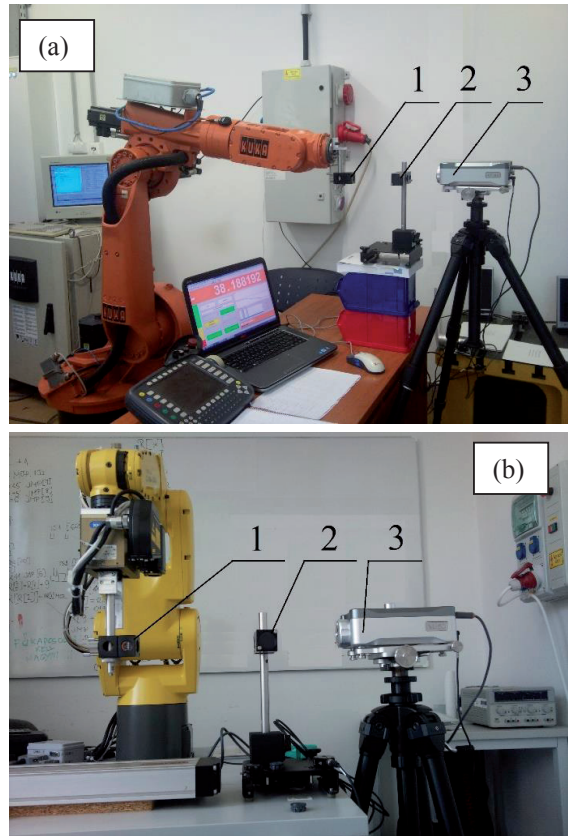

Fig. 4: Positioning measurement: (a) KUKA robot and (b) Fanuc robot.

Table 2: Starting joint angles of robots KUKA KR 15/2 and Fanuc LR Mate 200iC.

\begin{tabular}{|l|l|l|}
\hline \multicolumn{1}{|c|}{ Joint } & \multicolumn{1}{c|}{$\begin{array}{c}\text { KUKA } \\
\text { Joint angle["] }\end{array}$} & \multicolumn{1}{c|}{\begin{tabular}{c}
\multicolumn{1}{c|}{ Fanuc } \\
Joint angle[ [']
\end{tabular}} \\
\hline $\mathrm{J} 1$ & $-90^{\circ}$ & $0^{\circ}$ \\
\hline $\mathrm{J} 2$ & $-90^{\circ}$ & $0^{\circ}$ \\
\hline $\mathrm{J} 3$ & $95^{\circ}$ & $-14^{\circ}$ \\
\hline $\mathrm{J} 4$ & $0^{\circ}$ & $0^{\circ}$ \\
\hline $\mathrm{J} 5$ & $-5^{\circ}$ & $-76^{\circ}$ \\
\hline $\mathrm{J} 6$ & $0^{\circ}$ & $90^{\circ}$ \\
\hline
\end{tabular}

$X$ direction then secondly along $Y$ direction with $\pm 150 \mathrm{~mm}$ from the starting positions. In both cases the distance steps are $1 \mathrm{~mm}$ and the velocity of the motions was prescribed to $25 \mathrm{~mm} / \mathrm{s}$. The positions were measured by laser head and the errors are 
shown in Fig. 5 and Fig. 6 for motions in $X$ and $Y$ directions, respectively.

The positioning errors of the KUKA robot are denoted by green lines, while errors of the Fanuc robot are denoted by blue ones. In Fig. 5 and Fig. 6, the measurements in negative and in positive directions are given in a/ and b/ diagrams, respectively.

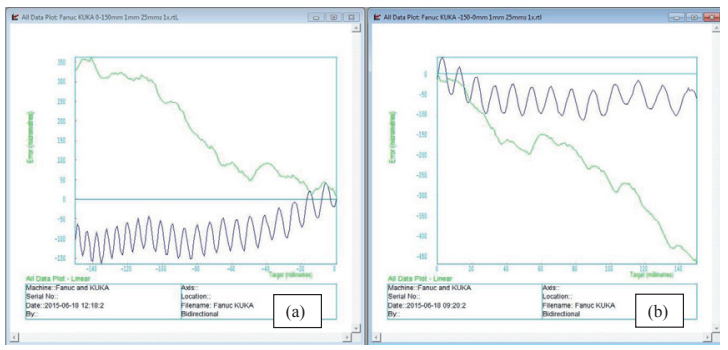

Fig. 5: Positioning errors (a) in negative $X$ direction, (b) in positive $X$ direction, where green and blue lines denote the KUKA and Fanuc robots, respectively.

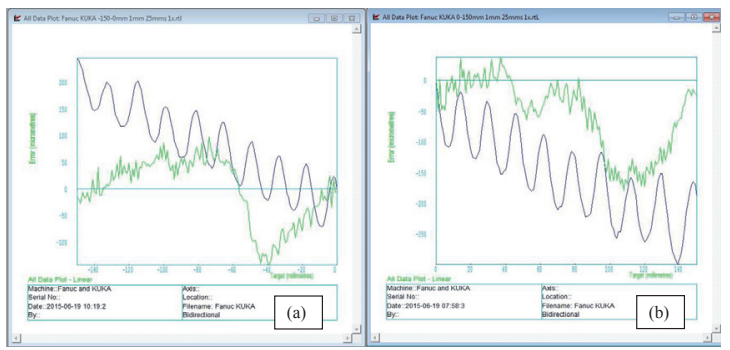

Fig. 6: Positioning errors (a) in negative $Y$ direction (b) in positive $Y$ direction, where green and blue lines denote the KUKA and Fanuc robots, respectively.

One can see that the positioning errors show increasing tendencies during the motion from the starting point in $\pm X$ directions (see Fig. 5). On the measured interval $(-150 \mathrm{~mm},+150 \mathrm{~mm})$ the KUKA robot performed about $+350 \mu \mathrm{m}$ and $-450 \mu \mathrm{m}$ maximal errors, while the Fanuc robot $-150 \mu \mathrm{m}$ and $-100 \mu \mathrm{m}$ errors. The measurements in $Y$ directions are shown in Fig. 6 where the KUKA robot produced about $-150 \mu \mathrm{m}$ maximal errors, while the Fanuc robot $+250 \mu \mathrm{m}$ and $-300 \mu \mathrm{m}$ errors. The diagrams for the KUKA show random oscillations, and almost regular oscillations can be seen for the Fanuc robot.

The above measurements were performed for different velocities and distance steps, and similar inaccuracies were obtained. Due to lack of space results are not shown here.

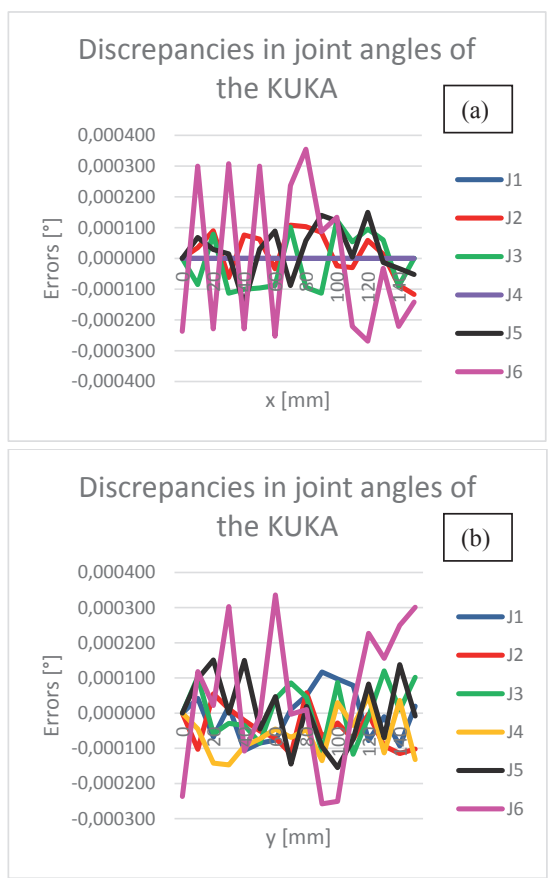

Fig. 7: Discrepancies in joint angles of KUKA robot comparing values provided by the controller and the theoretical formulae during the motions are (a) in X direction (b) in Y direction.

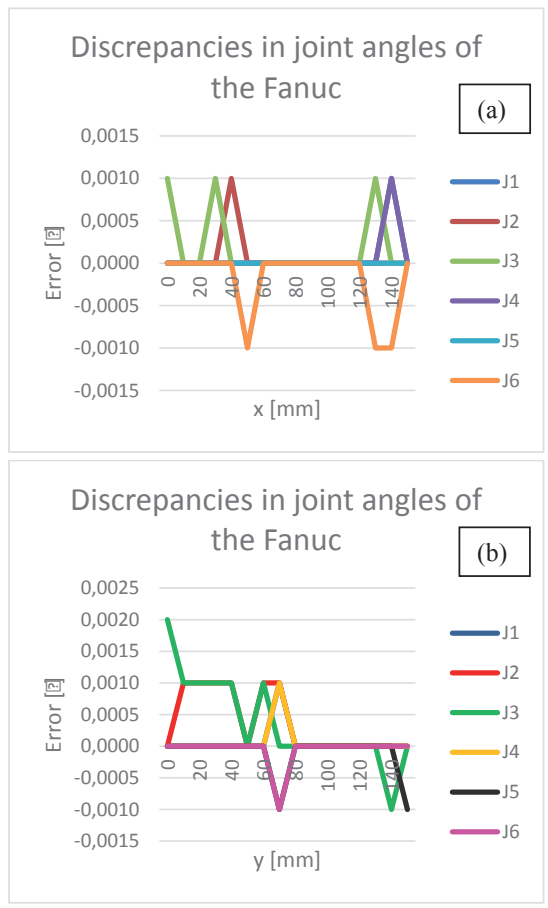

Fig. 8: Discrepancies in joint angles of Fanuc robot comparing values provided by the controller and the theoretical formulae during the motions are (a) in $X$ direction (b) in $Y$ direction. 
In order to investigate the sources of the positioning errors, the theoretical values of the joint angles are compared to the data provided by teaching boxes of the robots. The discrepancies of the two values are shown in Fig. 7 and Fig. 8 for the KUKA and Fanuc robots, respectively. The data of joint angles are given with six decimal precision by the controller of KUKA robot and three decimal precision by Fanuc robot. Results show that the errors in angles are practically negligible, since it may cause errors in positioning only a couple of $\mu \mathrm{m}$.

It is likely that the lion part of the positioning errors shown in Fig. 5 and Fig. 6 is resulted from the flexibility of the driving systems of the joints, which requires further investigation.

\section{Conclusions}

Positioning accuracies of two industrial robots have been analyzed in this paper. The positions of a robot KUKA KR 15/2 and a robot Fanuc LR Mate 200iC have been measured with Renishaw XL-80 type laser measurement system. The joint angles were evaluated by the help of analytical formulae, which were compared to angle values provided by the robot controllers. It was found that the discrepancies in joint angles are negligible.

It is shown that the positioning errors for the KUKA robot oscillate randomly, while almost regular oscillations can be seen for the Fanuc robot. Since the errors larger than $0.1 \mathrm{~mm}$ in positioning the investigated robots are not applicable for precise machining.

Further investigations are needed to determine the causes of the positioning errors.

\section{Acknowledgements \\ This research was carried out in the framework of the Center of Excellence of Mechatronics on Logistics at University of Miskolc.}

\section{References and notes}

[1] Virgala, I., Kelemen, M., Varga, M., Kuryło, P.: Analysing, Modeling and Simulation of Humanoid Robot Hand Motion. In: Procedia Engineering: Modelling of Mechanical and Mechtronic Systems MMaMS 2014: 25th-27th November 2014 High Tatras, Slovakia. Vol. 96(2014), p.489-499.-ISSN 1877 7058.

[2] Javier Andres, Luis Gracia, Josep Tornero: Implementation and testing of a CAM postprocessor for an industrial redundant workcell with evaluation of several fuzzified Redundancy Resolution Schemes, Robotics and ComputerIntegrated Manufacturing 28, 265-274, 2012.

[3] Fabricio Tadeu Paziani, Benedito Di Giacomo, Roberto Hideaki Tsunaki: Robot measuring form errors, Robotics and Computer-Integrated Manufacturing 25, 168-177, 2009.

[4] KUKA Roboter GmbH: Robot KR15/2 Technical Data, Augsburg, 1998.

[5] LR Mate 200iC Series \& R-30iA Mate Controller, 2009, http:// www.fanucrobotics.com/cmsmedia/datasheets/LR\%20 Mate\%20200iC\%20Series_10.pdf

[6] Spong, M., W. - Hutchinson, S. - Vidyasagar, M.: Robot Modeling and Control, John Wiley \& Sons, Inc. New York, USA 2005.

[7] RENISHAW: XL-80 Laser system training manual, 2000 .

[8] Portable laser measurement and calibration, 2013, http:// resources.renishaw.com/en/download/brochure-xl-80laser-system--59031

\section{Biographical notes}

Ákos Cservenák, is an MSc student at the Robert Bosch Department of Mechatronics in University of Miskolc, Hungary. He was born in Miskolc in 1991. He got BSc degree with honours in mechatronical engineering at the University of Miskolc in 2014. He started the master program in 2014 at the Robert Bosch Department of Mechatronics.

Tamás Szabó, is an Associated Professor at Robert Bosch Department of Mechatronics in University of Miskolc, Hungary. He was born in Tolcsva, Hungary in 1956. He got his mechanical engineering diploma at the Technical University for Heavy Industry in Miskolc, Hungary. He received his PhD degree in 1998 at the University of Miskolc. He is the head of the Robert Bosch Department of Mechatronics at the University of Miskolc. 